\title{
A Review of the Principles of Radiological Assessment of Skeletal Dysplasias
}

\author{
Yasemin Alanay1, Ralph S. Lachman2,3 \\ 1 Pediatric Genetics Unit, Department of Pediatrics, Faculty of Medicine, Acibadem University, Istanbul, Turkey \\ 2 Professor Emeritus of Radiological Sciences and Pediatrics, UCLA School of Medicine, Los Angeles, California, Clinical Professor, \\ Stanford University, Stanford CA, USA \\ 3International Skeletal Dysplasia Registry, Genetics Institute, Cedars-Sinai Medical Center, Los Angeles, California, USA
}

\begin{abstract}
There are more than 450 well-characterized skeletal dysplasias classified primarily on the basis of clinical, radiographic, and molecular criteria. In the latest 2010 revision of the Nosology and Classification of Genetic Skeletal Disorders, an increase from 372 to 456 disorders had occurred in the four years since the classification was last revisited in 2007. These entities in total represent about $5 \%$ of children with birth defects. An accurate diagnosis of a skeletal dysplasia is still based on detailed evaluation of clinical and radiographic las well as chondro-osseous] findings. Regardless of the specific diagnosis, skeletal dysplasias in general share clinical and radiological findings helping us to group them in several ways. This review aims to outline the diagnostic approach to disproportionate short stature with special emphasis on radiological findings.
\end{abstract}

Key words: Skeletal dysplasia, disproportionate short stature, radiology

Conflict of interest: None declared

Received: 12.08.2011

Accepted: 23.08 .2011

\section{Introduction}

Skeletal dysplasias are disorders associated with a generalized abnormality in the skeleton. Although individually rare, the overall birth incidence is estimated to be $1 / 5000$ live births (1). Today, there are more than 450 well-characterized skeletal dysplasias classified primarily on the basis of clinical, radiographic, and molecular criteria (2). Half a century ago, in the 1960s, individuals with disproportionate short stature were diagnosed either as achondroplasia (short-limbed dwarfism) or Morquio syndrome (short-trunked dwarfism). In time, delineation of numerous entities not fitting these two "disorders" led experts to come up with a systematic approach. The "International Nomenclature of Constitutional Diseases of Bone" group, since its first publication in 1970, has intermittently classified these disorders (1970-1977-19831992-2001-2005-2009) (3). In the 1970s, the categories were purely clinical and descriptive. This later evolved into a combination of clinical, radiological and molecular knowledge as the pathogenetic mechanisms of various entities have been revealed. In the latest 2010 revision of the Nosology and Classification of Genetic Skeletal Disorders, an increase from 372 to 456 disorders was noted in the four years since the classification was last revisited in $2007(2,4)$. Of these conditions, 316 are associated with one or more of 226 different genes. This increase reflects the 
continuing delineation of unique phenotypes among short stature conditions, which in aggregate represent about $5 \%$ of children with birth defects (1). Some of the increase has also been driven by technological improvements in our ability to define the molecular genetic basis of these conditions, which is now known for 316 of the disorders (215 in the prior revision), with defects in 226 (140 previously) different genes. Table 1 provides a list of main groups in the latest published classification (2).

In daily practice however, clinicians dealing with patients with short stature may be confused with the molecular listings. It is therefore important to remember that an accurate diagnosis of a skeletal dysplasia is still based on detailed evaluation of clinical and radiographic [as well as chondro-osseous] findings. This review aims to outline the diagnostic approach to disproportionate short stature with special emphasis on radiological findings.

\section{Clinical Evaluation}

The accurate history regarding time of onset of short stature is essential prior to physical examination. Among the nearly 400 skeletal dysplasias, 100 or so have prenatal onset, while others may only present either as newborns or beyond 2 to 3 years of age (5). Individuals with disproportionate short stature are likely to be affected by a skeletal dysplasia. However, the abnormal proportions may not be readily recognizable. Therefore, whenever an individual presents with short stature, it is essential to measure body proportions. This should be done keeping in mind that some generalized bone mineralization abnormalities such as osteogenesis imperfects (OI), some osteosclerotic disorders, and hypophosphatasia may present with near normal proportions.

Anthropometric measurements such as upper/lower segment (U/L) ratio, sitting height, and arm span are routinely measured when a patient with short stature is evaluated. Sitting height is the measurement of head and trunk, and may be difficult to measure accurately due to the need of special equipment. The lower segment, however, is easier to measure (from symphysis pubis towards the floor medially to the heel). The upper segment can then be easily calculated by subtracting the lower segment from total height. Upper and lower segment measurements can be made in a standing or supine position. The mentioned ratios change with age. $U / L$ ratio is 1.7 in the newborn; approximately 1.0 between ages $2-8$ years; 0.95 as an adult. A short statured patient with short trunk will have decreased a U/L ratio, while an individual with normal trunk and relatively short limbs will have an increased $U / L$ ratio (6).
Clinical evaluation also includes description of the limb involvement. Depending on the primarily involved segment of the limb, the condition can be described as rhizomelic (humerus and femur), mesomelic (radius, ulna, tibia and fibula) and acromelic (hands and feet). These descriptions help in differential diagnosis. It is noteworthy that a careful examination by an experienced clinical geneticist can sometimes narrow the list of dysmorphological entities to be considered even before the skeletal radiographs are analyzed (7).

Other clinical assessments such as immunological /hematological data as well as hair quality, cleft palate, eye abnormalities (myopia) and even internal organ abnormalities (cystic kidneys, hepatosplenomegaly) are important in skeletal dysplasia evaluation.

After obtaining a thorough family history, constructing a detailed pedigree and performing clinical examination, radiological assessment is likely to close the case in most skeletal dysplasias as many have distinctive radiological features in growing bones.

\section{Radiological Assessment}

Before giving details of the stepwise radiographic analysis for skeletal dysplasias; we would like to emphasize that a complete "genetic skeletal survey" is not necessary in patients with proportionate short stature, in which the differential diagnosis consists of constitutional delay, familial short stature, a small group of endocrinopathies and some dysmorphic syndromes. Their initial imaging assessment may warrant a left hand and wrist radiograph for bone age determination. This will protect children from unnecessary radiation exposure.

The "genetic skeletal survey" should include anteroposterior (AP), and lateral views of the skull, AP and lateral views of the entire spine, and AP views of the pelvis and all four extremities, with separate AP views of the hands and feet [A lateral view of the knee can be helpful to diagnose a recessive form of multiple epiphyseal dysplasia (MED) associated with multilayered patella] (7). In adult patients, it is mandatory to try to obtain prepubertal skeletal radiographs. Once the epiphyses have fused to the metaphyses, diagnosis may be very difficult. After obtaining the radiographs, a three-step assessment will be helpful in trying to make a specific diagnosis.

Step I (Assessment of Disproportion): An assessment of disproportion similar to the one made clinically is repeated looking at the radiographs. A quick look at the spine will readily help decide if there is platyspondyly leading to short-trunked disproportion. Similarly, looking at the 
Alanay $Y$ et al.

Radiological Evaluation of Skeletal Dysplasias

Table 1. Osteochondrodysplasias (Nosology and Classification of Genetic Skeletal Disorders: 2010 Revision)

\begin{tabular}{|c|c|c|c|c|c|}
\hline Name of Disorder & Inheritance & MIM No. & Locus & Gene & Protein \\
\hline \multicolumn{6}{|l|}{ 1. FGFR3 group } \\
\hline Thanatophoric dysplasia type 1 (TD1) & $A D$ & 187600 & $4 p 16.3$ & FGFR3 & FGFR3 \\
\hline Thanatophoric dysplasia type 2 (TD2) & $A D$ & 187601 & $4 p 16.3$ & FGFR3 & FGFR3 \\
\hline $\begin{array}{l}\text { SADDAN } \\
\text { (severe achondroplasia-developmental delay- acanthosis nigricans) }\end{array}$ & $A D$ & $\begin{array}{c}\text { See } \\
134934\end{array}$ & $4 p 16.3$ & FGFR3 & FGFR3 \\
\hline Achondroplasia & $A D$ & 100800 & $4 p 16.3$ & FGFR3 & FGFR3 \\
\hline Hypochondroplasia & $A D$ & 146000 & $4 p 16.3$ & FGFR3 & FGFR3 \\
\hline Hypochondroplasia-like dysplasia & $A D, S P$ & & & & \\
\hline Camptodactyly, tall stature, and hearing loss syndrome (CATSHL & L) $\mathrm{AD}$ & 187600 & $4 p 16.3$ & FGFR3 & FGFR3 \\
\hline
\end{tabular}

\section{Type 2 collagen group}

Achondrogenesis type 2 (ACG2; Langer-Saldino)

Platyspondylic dysplasia, Torrance type

Hypochondrogenesis

Spondyloepiphyseal dysplasia congenital (SEDC)

Spondyloepimetaphyseal dysplasia

(SEMD) Strudwick type

Kniest dysplasia

Spondyloperipheral dysplasia

Mild SED with premature onset arthrosis

SED with metatarsal shortening (formerly Czech dysplasia)

Stickler syndrome type 1

Stickler-like syndrome

A

$A D$

$200610 \quad 12 q 13.1$

COL2A1

Type 2 collagen

$A D$

151210

$12 q 13.1$

COL2A1

Type 2 collagen

$\mathrm{AD}$

200610

$12 q 13.1$

COL2A1

Type 2 collagen

$A D$

183900

COL2A1

Type 2 collagen

AD

184250

$12 q 13.1$

COL2A1

Type 2 collagen

$A D$

$156550 \quad 12 q 13.1$

COL2A1

Type 2 collagen

$A D$

271700

12q13.1

COL2A1

Type 2 collagen

$A D$

$12 q 13.1$

COL2A1

Type 2 collagen

$A D$

$609162 \quad 12 q 13.1$

COL2A1

Type 2 collagen

$A D$

108300

$12 q 13.1$

COL2A1

Type 2 collagen

\section{Type 11 collagen group}

Stickler syndrome type 2

$A D$

604841

Marshall syndrome

Fibrochondrogenesis

Otospondylomegaepiphyseal dysplasia

(OSMED), recessive type

Otospondylomegaepiphyseal dysplasia

(OSMED), dominant type (Weissenbacher-

Zweymüller syndrome, Stickler syndrome type 3)

\section{Sulphation disorders group}

Achondrogenesis type 1B (ACG1B)

Atelosteogenesis type 2(A02)

Diastrophic dysplasia (DTD)

MED, autosomal recessive type (rMED; EDM4)

SEMD, PAPSS2 type
$A D$

AR

154780

228520

AR

215150

$A D$

2151

215150

$6 \mathrm{p} 21.3$

AR

AR

AR

22260

60097

$5 q 32-33$

AR

226900

$5 q 32-33$

AR
$603005 \quad 10 q 23-q 24$
DTDST

DTDST

DTDST

DTDST

COL11A1

alpha-1 chain

COL11A1 alpha-1 chain

COL11A2

Type 11 collagen alpha-2 chain

Type 11 collagen alpha-2 chain

SLC26A2

sulfate transporter

SLC26A2 sulfate transporter

SLC26A2

sulfate transporter

SLC26A2

sulfate transporter

PAPS-Synthetase 2 
Table 1. (continued)

Chondrodysplasia with congenital joint dislocations, CHST3 type (recessive Larsen syndrome)

Ehlers-Danlos syndrome, CHST14 type ("musculo-skeletal variant")

\section{Perlecan group}

Dyssegmental dysplasia, Silverman- Handmaker type Dyssegmental dysplasia, Roland-Desbuqois

Schwartz-Jampel syndrome (myotonic chondrodystrophy)

\section{Aggrecan group}

SED, Kimberley type

SEMD, Aggrecan type

Familial osteochondritis dissecans

\section{Filamin group and related disorders}

Frontometaphyseal dysplasia

Osteodysplasty Melnick-Needles

Otopalatodigital syndrome type 1 (OPD1)

Otopalatodigital syndrome type 2 (OPD2)

Atelosteogenesis type 1 (A01)

Atelosteogenesis type 3 (A03)

Larsen syndrome

Spondylo-carpal-tarsal dysplasia

Franck-ter-Haar syndrome

Serpentine fibula-polycystic kidney syndrome

\section{TRPV4 group}

Metatropic dysplasia

Spondyloepimetaphyseal dysplasia, Maroteaux type (Pseudo-Morquio syndrome type 2)

Spondylometaphyseal dysplasia, Kozlowski type

Brachyolmia, autosomal dominant type

Familial digital arthropathy with brachydactyly

\section{Short-rib dysplasias (with or without polydactyly) group}

Chondroectodermal dysplasia (Ellis-van Creveld)

SRP type 1/3 (Saldino-Noonan/Verma-Naumoff)

SRP type 1/3 (Saldino-Noonan/Verma-Naumoff)

SRP type 1/3 (Saldino-Noonan/Verma-Naumoff)

SRP type 2 (Majewski)

SRP type 4 (Beemer)

$\begin{array}{lllll}\text { AR } & 608637 & 10 \mathrm{q} 22.1 & \text { CHST3 } & \text { C } \\ \text { AR } & 601776 & 15 q 14 & \text { CHST14 } & \text { Ca }\end{array}$

Carbohydrate sulfotransferase 3 ; Chondroitin 6-sulfotransferase

Carbohydrate sulfotransferase 14; dermatan 4-sulfotransferase

$\begin{array}{lllll}\text { AR } & 224410 & 1 \mathrm{q} 36-34 & \text { PLC (HSPG2) } & \text { Perlecan } \\ \text { AR } & 224400 & 1 \mathrm{q} 36-34 & \text { PLC (HSPG2) } & \text { Perlecan } \\ \text { AR } & 255800 & 1 \mathrm{q} 36-34 & \text { PLC (HSPG2) } & \text { Perlecan }\end{array}$

$A D$

AR

$A D$

608361

$15 q 26$

612813

165800

$15 q 26$

$15 q 26$

$\mathrm{Xq} 28$

$\mathrm{Xq} 28$

$\mathrm{Xq} 28$

$\mathrm{Xq} 28$

$3 p 14.3$

$3 p 14.3$

$3 p 14.3$

$3 p 14.3$

$5 q 35.1$

249420

AR

$A D$ ?

600330

156530

$12 q 24.1$

$A D$

$A D$

184095

12q24.1

184252

12q24. 1

$A D$

113500

$12 q 24.1$

606835

$12 q 24$.

$A D$

AR

AR

AR

263510

$3 q 25.33$

AR $\quad 263510$

AR

263520

AR
AGC1

AGC1

AGC1

FLNA

FLNA

FLNA

FLNA

FLNB

FLNB

FLNB

FLNB

SH3PXD28

\section{Aggrecan}

Aggrecan

Aggrecan

Filamin A

Filamin A

Filamin A

Filamin A

Filamin B

Filamin B

Filamin B

Filamin B

TKS4
TRPV4 Transient receptor potential cation channel, subfamily V, member 4

TRPV4 Transient receptor potential cation channel, subfamily V, member 4

TRPV4 Transient receptor potential cation channel, subfamily V, member 4

TRPV4 Transient receptor potential cation channel, subfamily V, member 4

TRPV4 Transient receptor potential cation channel, subfamily V, member 4

EVC1

EVC2

EvC gene 1

EvC gene 2

Dynein, cytoplasmic 2 heavy chain 1

Intraflagellar transport 80 (homolog of)

NEK1

\section{Nima related kinase 1}


Alanay $Y$ et al.

Radiological Evaluation of Skeletal Dysplasias

Table 1. (continued)

Oral-Facial-Digital syndrome type 4 (Mohr-Majewski)

Asphyxiating thoracic dysplasia (ATD; Jeune)

Asphyxiating thoracic dysplasia (ATD; Jeune)

Asphyxiating thoracic dysplasia (ATD; Jeune)

Thoracolaryngopelvic dysplasia (Barnes)

10. Multiple epiphyseal dysplasia and pseudoachondroplasia group

Pseudoachondroplasia (PSACH)

Multiple epiphyseal dysplasia (MED) type 1 (EDM1)

Multiple epiphyseal dysplasia (MED) type 2 (EDM2)

Multiple epiphyseal dysplasia (MED) type 3 (EDM3)

Multiple epiphyseal dysplasia (MED) type 5 (EDM5)

Multiple epiphyseal dysplasia (MED) type 6 (EDM6)

Multiple epiphyseal dysplasia (MED), other types

Stickler syndrome, recessive type

Familial hip dysplasia (Beukes)

Multiple epiphyseal dysplasia with microcephaly and nystagmus (Lowry-Wood)

\section{Metaphyseal dysplasias}

Metaphyseal dysplasia, Schmid type (MCS)

Cartilage-hair-hypoplasia

(CHH; metaphyseal dysplasia, McKusick type)

Metaphyseal dysplasia, Jansen type

Eiken dysplasia

Metaphyseal dysplasia with pancreatic

insufficiency and cyclic neutropenia

(Shwachman-Bodian-Diamond syndrome, SBDS)

Metaphyseal anadysplasia type 1

Metaphyseal anadysplasia type 2

Metaphyseal dysplasia, Spahr type

Metaphyseal acroscyphodysplasia (various types)

Genochondromatosis (type1/type 2)

Metaphyseal chondromatosis with D-2-hydroxyglutaric aciduria

\section{Spondylometaphyseal dysplasias (SMD)}

Spondyloenchondrodysplasia (SPENCD)

Odontochondrodysplasia (ODCD)

Spondylometaphyseal dysplasia Kozlowski type

Spondylometaphyseal dysplasia, Sutcliffe/corner fracture type

SMD with severe genu valgum

SMD with cone-rod dystrophy

SMD with retinal degeneration, axial type

Dysspondyloenchondromatosis

Cheiro-spondyloenchondromatosis

\begin{tabular}{|c|c|c|c|c|}
\hline AR & 258860 & & & \\
\hline AR & 208500 & $11 q 22.3$ & DYNC2H1 & $\begin{array}{c}\text { Dynein, cytoplasmic } 2, \\
\text { heavy chain } 1\end{array}$ \\
\hline AR & 208500 & $3 q 25.33$ & IFT80 & $\begin{array}{l}\text { Intraflagellar transport } \\
80 \text { (homolog of) }\end{array}$ \\
\hline
\end{tabular}

AR $\quad 208500$

AD $\quad 187760$

$A D$

177170

COMP

COMP

$A D$

132400

$19 \mathrm{p} 13.1$

COMP

COL9A2

$600204 \quad 1 \mathrm{p} 32.2-33$

COL9A3

$A D$

60096

$20 q 13.3$

COL9A3

$A D$

607078

2p23-24

MATN3

$A D$

$6 q 13$

COL9A1

\section{COMP}

Collagen 9 alpha-2 chain

Collagen 9 alpha-3 chain

Matrilin 3

Collagen 9 alpha-1 chain

AR

120210

$6 q 13$

COL9A1

Collagen 9 alpha- 1 chain

$A D \quad 142669 \quad 4 q 35$

AR $\quad 226960$
$A D$

AR

$A D$

$A R$

AR

156500

250250

156400

600002

260400

3p22-22.1

$A D, A R$

$A R$

AR

AR

$A D / S P$

$\mathrm{AR} / \mathrm{SP}$

309645

250215

137360

271550

AR

AR

271550

$19 p 13.2$

ACP5
$7 q 11$

Collagen 10 alpha- 1 chain RNA component of RNAse $\mathrm{H}$

PTH/PTHrP receptor 1 PTH/PTHrP receptor 1 SBDS gene, function unclear

PTHR1

SBDS

MMP13

Matrix metalloproteinase 13

20q13.12 MMP9

Matrix metalloproteinase 9
$A D \quad 184252$

AD $\quad 184255$

AD $\quad 184253$

AR $\quad 608940$

AR $\quad 602271$ 


\begin{tabular}{|c|c|c|c|c|c|}
\hline \multicolumn{6}{|l|}{ 13. Spondylo-epi(-meta)physeal dysplasias (SE(M)D) } \\
\hline Dyggve-Melchior-Clausen dysplasia (DMC) & $A R$ & $223800 \quad 1$ & $18 q 12-21.1$ & DYM & Dymeclin \\
\hline Immuno-osseous dysplasia (Schimke) & AR & 242900 & $2 q 34-36$ & SMARCAL1 & $\begin{array}{l}\text { SWI/SNF-related } \\
\text { regulator of chromatin } \\
\text { subfamily A-like protein } 1\end{array}$ \\
\hline SED Wolcott-Rallison type & AR & 226980 & $2 p 12$ & EIF2AK3 & $\begin{array}{l}\text { Translation initiation } f \\
\text { actor 2-alpha kinase-3 }\end{array}$ \\
\hline SEMD Matrilin type & $A R$ & 608728 & $2 p 23-p 24$ & MATN3 & Matrilin 3 \\
\hline SEMD Missouri type & $A D$ & 602111 & $11 q 22.3$ & MMP13 & Matrix metalloproteinase 13 \\
\hline Metatropic dysplasia (various forms) & $\mathrm{AD} / \mathrm{AR}$ & 156530 & & & \\
\hline SED tarda, X-linked (SED-XL) & $\mathrm{XLR}$ & 313400 & Xp22 & SEDL & Sedlin \\
\hline SPONASTRIME dysplasia & AR & 271510 & & & \\
\hline SEMD short limb - abnormal calcification type & AR & 271665 & $1 q 23$ & DDR2 & $\begin{array}{l}\text { Discoidin domain receptor } \\
\text { family, member2 }\end{array}$ \\
\hline SEMD with joint laxity (SEMD-JL) Beighton type & $A R$ & 271640 & & & \\
\hline Spondylo-megaepiphyseal-metaphyseal dysplasia (SMMD) & AR & 613330 & $4 p 16.1$ & NKX3 & NK3 Homeobox \\
\hline Spondylodysplastic Ehlers-Danlos syndrome & AR & 271510 & $11 p .11 .2$ & SLC39A13 & Zinc transporter ZIP13 \\
\hline $\begin{array}{l}\text { SEMD with joint laxity (SEMD-JL) leptodactylic or } \\
\text { Hall type }\end{array}$ & $A D$ & 603546 & & & \\
\hline Platyspondyly (brachyolmia) with amelogenesis imperfecta & $A R$ & 601216 & & & \\
\hline Late onset SED, autosomal recessive type & $A R$ & 609223 & & & \\
\hline Brachyolmia, Hobaek, and Toledo types & AR & 271530,271630 & & & \\
\hline \multicolumn{6}{|l|}{ 14. Severe spondylodysplastic dysplasias } \\
\hline Achondrogenesis type $1 \mathrm{~A}$ (ACG1A) & $A R$ & 200600 & $14 q 32.12$ & TRIP11 & $\begin{array}{l}\text { Golgi-microtubule-assocated } \\
\text { protein, 210-kDa; GMAP210 }\end{array}$ \\
\hline SMD Sedaghatian type & AR & 250220 & & & \\
\hline Severe SMD Sedaghatian-like & AR & & $7 q 11$ & SBDS & SBDS gene, function still unclear \\
\hline Opsismodysplasia & AR & 258480 & & & \\
\hline Schneckenbecken dysplasia & AR & 269250 & $1 \mathrm{p} 31.3$ & SLC35D1 & $\begin{array}{c}\text { Solute carrier family } 35 \\
\text { member D1; UDP-glucuronic } \\
\text { acid/UDP-N-acetylgalactosamine } \\
\text { dual trasporter }\end{array}$ \\
\hline \multicolumn{6}{|l|}{ 15. Acromelic dysplasias } \\
\hline Trichorhinophalangeal dysplasia types $1 / 3$ & $A D$ & $\begin{array}{l}190350 \\
190351\end{array}$ & $8 q 24$ & TRPS1 & Zinc finger transcription factor \\
\hline Trichorhinophalangeal dysplasia type 2 (Langer- Giedion) & $A D$ & 150230 & $8 q 24$ & $\begin{array}{l}\text { TRPS1 } \\
\text { EXT1 }\end{array}$ & $\begin{array}{l}\text { Zinc finger transcription factor } \\
\text { Exostosin } 1\end{array}$ \\
\hline Acrocapitofemoral dysplasia & $A R$ & 607778 & $2 q 33-q 35$ & $\mathrm{IHH}$ & Indian hedgehog \\
\hline Cranioectodermal dysplasia (Levin-Sensenbrenner) type 1 & AR & 218330 & & & \\
\hline Cranioectodermal dysplasia (Levin-Sensenbrenner) type 2 & AR & 613610 & $2 \mathrm{p} 24.1$ & WDR35 & WD repeat-containig protein 35 \\
\hline Geleophysic dysplasia & $A R$ & 231050 & $9 q 34.2$ & ADAMTSL2 & ADAMTS-like protein 2 \\
\hline Geleophysic dysplasia, other types & $A R$ & & & & \\
\hline Acromicric dysplasia & $A D$ & 102370 & & & \\
\hline Acrodysostosis & $A D$ & 101800 & & & \\
\hline Angel-shaped phalangoepiphyseal dysplasia (ASPED) & $A D$ & 105835 & & & \\
\hline Acrolaryngeal dysplasia & $A D$ & & & & \\
\hline Craniofacial conodysplasia & $A D$ & & & & \\
\hline Familial digital arthropathy with brachydactyly & $A D$ & 606835 & & & \\
\hline Saldino-Mainzer dysplasia & AR & 266920 & & & \\
\hline
\end{tabular}


Alanay $Y$ et al.

Radiological Evaluation of Skeletal Dysplasias

Table 1. (continued)

\section{Acromesomelic dysplasias}

Acromesomelic dysplasia type Maroteaux

Grebe dysplasia

Fibular hypoplasia and complex brachydactyly (Du Pan)

AR

602875

9p13-12

NPR2

Natriuretic peptide receptor 2

Hurar hypoplasia and complex brachydactyly (Du Pan)

AR

200700

$20 q 11.2$

GDF5

Growth and differentiation factor 5

Acromesomelic dysplasia with genital anomalies

AR

228900

$20 q 11.2$

GDF5

Growth and differentiation factor 5

Acromesomelic dysplasia, Osebold-Remondini type

609441

$4 q 23-24$

BMPR1B

Bone morphogenetic protein receptor 1B

$A D$

112910

\section{Mesomelic and rhizo-mesomelic dysplasias}

Dyschondrosteosis (Leri-Weill)

Langer type (homozygous dyschondrosteosis)

Robinow syndrome, recessive type

Robinow syndrome, dominant type

Mesomelic dysplasia, Korean type

Mesomelic dysplasia, Kantaputra type

Mesomelic dysplasia, Nievergelt type

Mesomelic dysplasia, Kozlowski-Reardon type

Mesomelic dysplasia with acral synostoses

(Verloes-David-Pfeiffer type)

$\begin{array}{ccc}\begin{array}{c}\text { Pseudo-AD } \\ \text { Pseudo-AR }\end{array} & 247300 & \text { Xpter-p22.32 } \\ \text { AR } & 268310 & \text { Xpter-p22.32 } \\ & & \\ \text { AD } 22 \\ \text { AD } & 180700 & \\ \text { AD } & 156232 & 2 q 24-32 \\ \text { AD } & 163400 & \\ \text { AR } & 249710 & \\ \text { AD } & 600383 & 8 q 13\end{array}$

SHOX

SHOX

ROR2

Short stature - homeobox gene

Short stature - homeobox gene

Receptor tyrosine kinase-like orphan receptor 2

Mesomelic dysplasia, Savarirayan type

605274

(Triangular Tibia-Fibular Aplasia)

\section{Bent bones dysplasias}

Campomelic dysplasia (CD)

Stüve-Wiedemann dysplasia

$A D$

$114290 \quad 17 q 24.3-25.1 \quad$ SOX9

AR

60155

$5 p 13.1$

LIFR

Cumming syndrome

211890

211350

Kyphomelic dysplasia, several forms

Bent bones at birth can be seen in a variety of conditions, including Antley-Bixler syndrome, cartilage-hair hypoplasia, hypophosphatasia, osteogenesis imperfecta, dyssegmental dysplasia, and others

\section{Slender bone dysplasia Group}

3-M syndrome (3M1)

3-M syndrome (3M2)

Kenny-Caffey dysplasia type 1

Kenny-Caffey dysplasia type 2

Microcephalic osteodysplastic primordial dwarfism type 1/3 (MOPD1)

Microcephalic osteodysplastic primordial dwarfism type 2 (MOPD2; Majewski type)

Microcephalic osteodysplastic dysplasia,

Saul-Wilson type

IMAGE syndrome (Intrauterine Growth Retardation, Metaphyseal Dysplasia, Adrenal Hypoplasia, and Genital Anomalies)

$\begin{array}{ccccc}\text { AR } & 273750 & 6 \mathrm{p} 21.1 & \text { CUL7 } & \text { Cullin 7 } \\ \text { AR } & 619921 & 2 q 35 & \text { PBSL1 } & \text { Obscurin-like 1 } \\ \text { AR } & 244460 & 1 q 42-q 43 & \text { TBCE } & \text { tubulin-specific chaperone E } \\ \text { AD } & 127000 & & & \\ \text { AR } & 210710 & 2 q & & \\ & & & & \\ \text { AR } & 210720 & 21 q & \text { PCNT2 } & \text { Pericentrin 2 }\end{array}$

AR

XL/AD $\quad 300290$
SRY-box 9

Heparan sulfatase

6-0-endosulfatase 1 and solute carrier organic anion transporter family member $5 \mathrm{~A} 1$

Leukemia inhibitory factor receptor 
Alanay $Y$ et al.

Radiological Evaluation of Skeletal Dysplasias

\begin{tabular}{lllll} 
Table 1. (continued) & & & & \\
\hline Osteocraniostenosis & SP & 602361 & & \\
Hallermann-Streiff syndrome & AR & 234100 & & \\
20. Dysplasias with multiple joint dislocations & & & & \\
$\begin{array}{l}\text { Desbuquois dysplasia (with accessory } \\
\text { ossification center in digit 2) }\end{array}$ & AR & 251450 & $17 q 25.3$ & CANT1 \\
$\begin{array}{l}\text { Desbuquois dysplasia with short metacarpals and } \\
\text { elongated phalanges) }\end{array}$ & AR & 251450 & $17 q 25.3$ & CANT1 \\
$\begin{array}{l}\text { DEsbuquois dysplasia (other variants with or without } \\
\text { accessory ossification center) }\end{array}$ & AR & & & \\
$\begin{array}{l}\text { Pseudodiastrophic dysplasia } \\
\text { Modified and reproduced from Warman ML et al. Nosology and Classification of Genetic Skeletal Disorders: 2010 Revision. Am J Med Genet 155A:943-968. }\end{array}$
\end{tabular}

extremities may help defining rhizomelia, mesomelia, and acromelia. It should be noted that these descriptive terms of limb segments may be more correct radiologically as the clinical visualization is accentuated by skin folds or other tissues rather than the length of the underlying bone. Rhizomelic chondrodysplasia punctata (CDP) is a good example of a rhizomelic skeletal dysplasia diagnosed with the additional radiological findings of punctate calcifications (stippling) and coronal clefted vertebrae (Figure 1). Mesomelia alone will suggest a long heterogeneous differential diagnosis list of mesomelic dysplasias. Presence of acromelia is important to recognize, as it may be an isolated finding. Presence of isolated acromelia may suggest skeletal dysplasias such as acromicric dysplasia, acrodysostosis, geleophysic dysplasia or nonskeletal dysplasias such as the brachydactylies. Brachydactyly type E, characterized by a short fourth metacarpal bone may support clinical or laboratory findings in Turner syndrome and pseudohypoparathyroidism, respectively. The absence of proportional acromelic shortening is also very important to remember in spondyloepiphyseal dysplasia congenita (and most forms of type II collagenopathies) (7).

Step II (Assessment of Epiphyseal/Metaphyseal/ Diaphyseal Ossification): Abnormal development of epiphyses, metaphyses, and diaphyses has given rise to the original nomenclature using those site names (Figure 2). An overall look at the radiological survey will suggest epiphyseal dysplasias by the presence of very small (delayed ossification) and/or irregularly ossified epiphyses (Figure 3a). If the metaphyses are widened, flared, and/or irregular, the diagnosis of a form of metaphyseal dysplasia is established (Figure 3b, 3c and Figure 4). Diaphyseal dysplasia is present when there is diaphyseal widening and/or cortical thickening or marrow space expansion or restriction. Isolated vertebral involvement without changes in the growth plate region in a patient with short-trunked

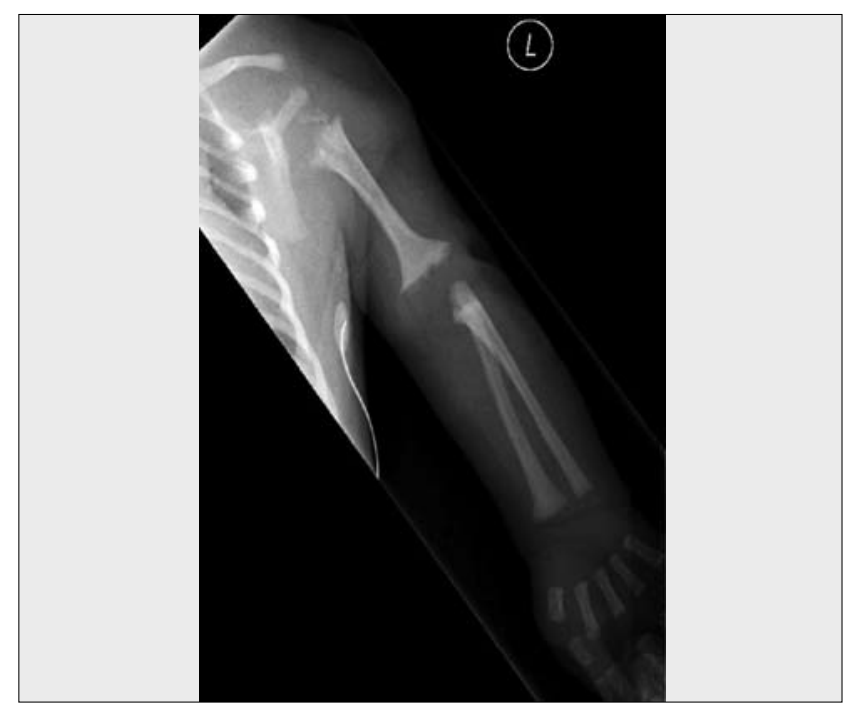

Figure 1. Rhizomelic chondrodysplasia punctata. Note shortness of humerus (rhizomelia and punctate calcifications in proximal humerus)

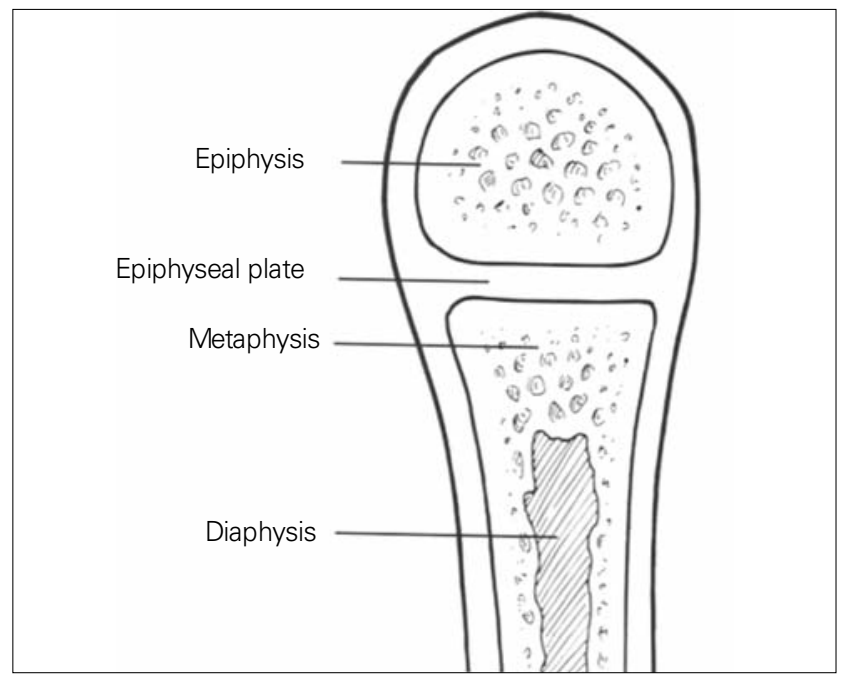

Figure 2. Key areas of the growing bone 
short stature should suggest brachyolmia (Figure 5a and 5b). Figure 3 helps to combine the aforementioned skeletal involvement, such as forms of spondyloepiphyseal dysplasia and the group of spondylo-epi-(meta)-physeal
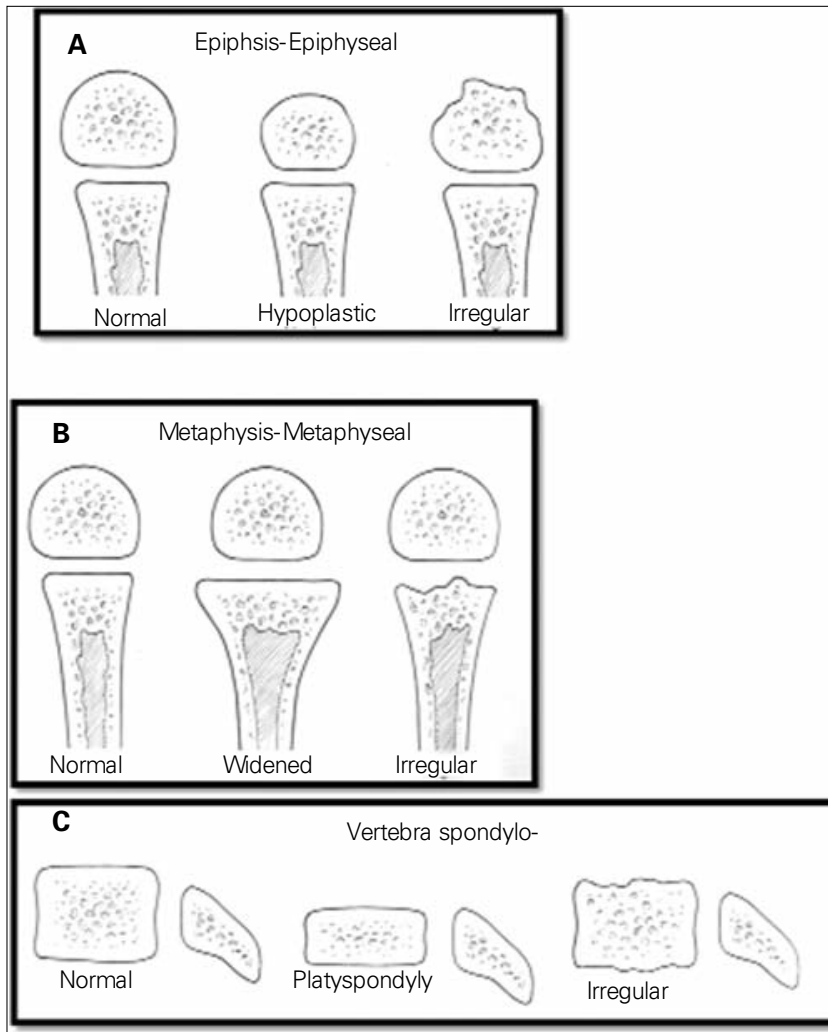

Figure 3. a,b,c. Radiographic manifestations of the dysplasias

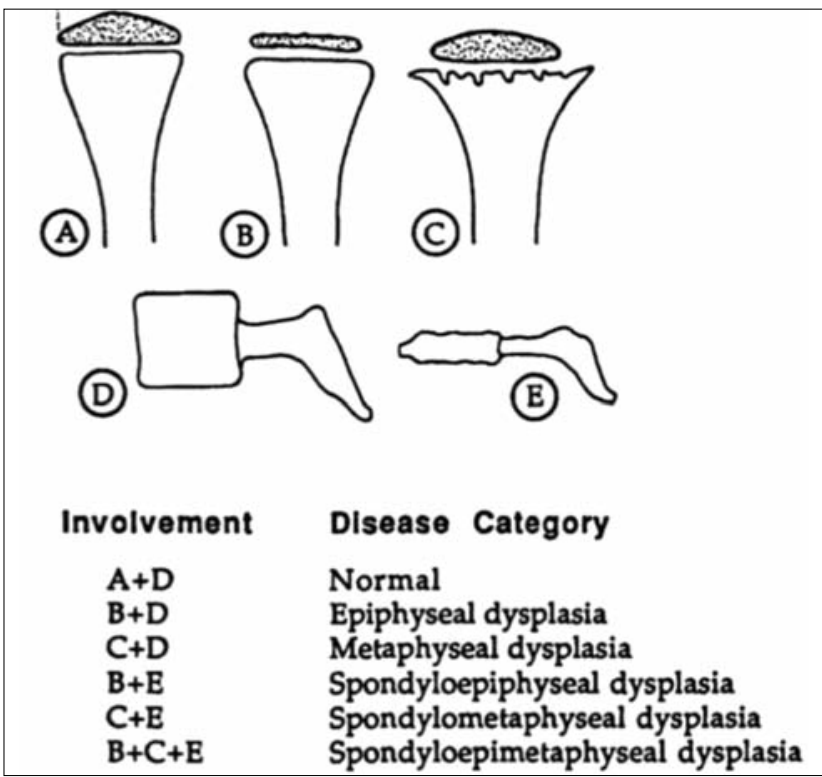

Figure 4. Radiographic abnormalities helpful in classification of skeletal dysplasias



Figure 5. a,b. Brachyolmia. Note platyspondyly and overfaced pedicles

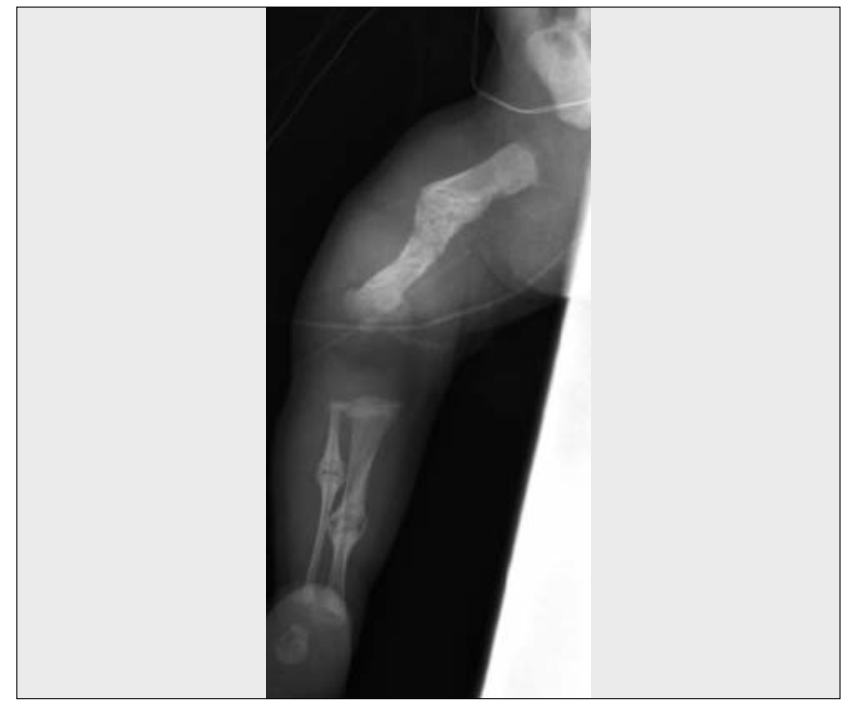

Figure 6. Fractures in Osteogenesis Imperfecta

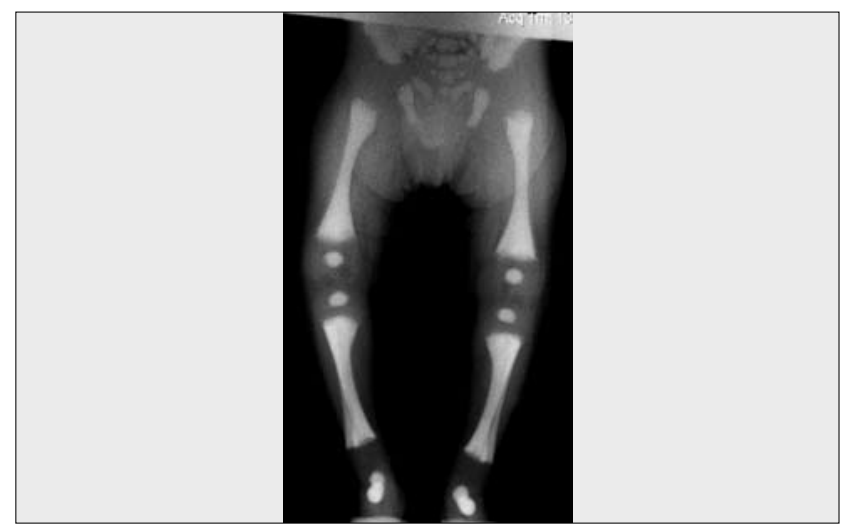

Figure 7. Osteopetrosis, generalized osteosclerosis 
dysplasias [SE(M)Ds]. Fractures can be seen in all types of Ol (Figure 6), osteosclerotic disorders including osteopetrosis (Figure 7) and severe hypophosphatasia (Figure 8) (7).

Following the evaluation of limb segments and the epiphyseal growth plate, focus on all the skeletal structures available in the genetic skeletal survey is mandatory to recognize a well-described skeletal dysplasia from a previous broad categorization into a specific group. This precise evaluation will include a search for pathognomonic findings, such as snail-shaped iliac bones of Schneckenbecken dysplasia (Figure 9), "lacy" appearance of iliac crest in Dyygve-Melchior-Clausen syndrome

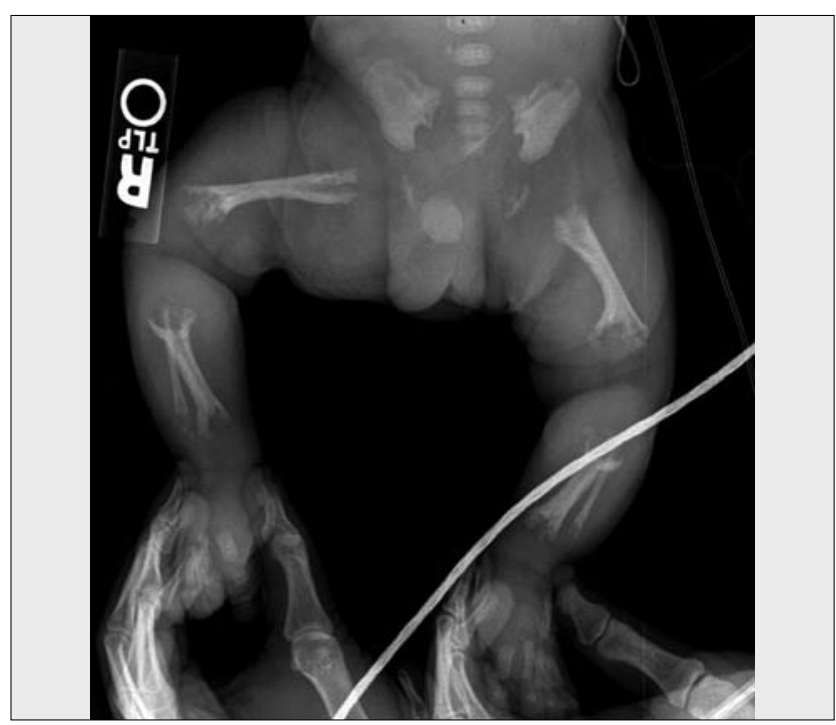

Figure 8. Infantile hypophosphatasia

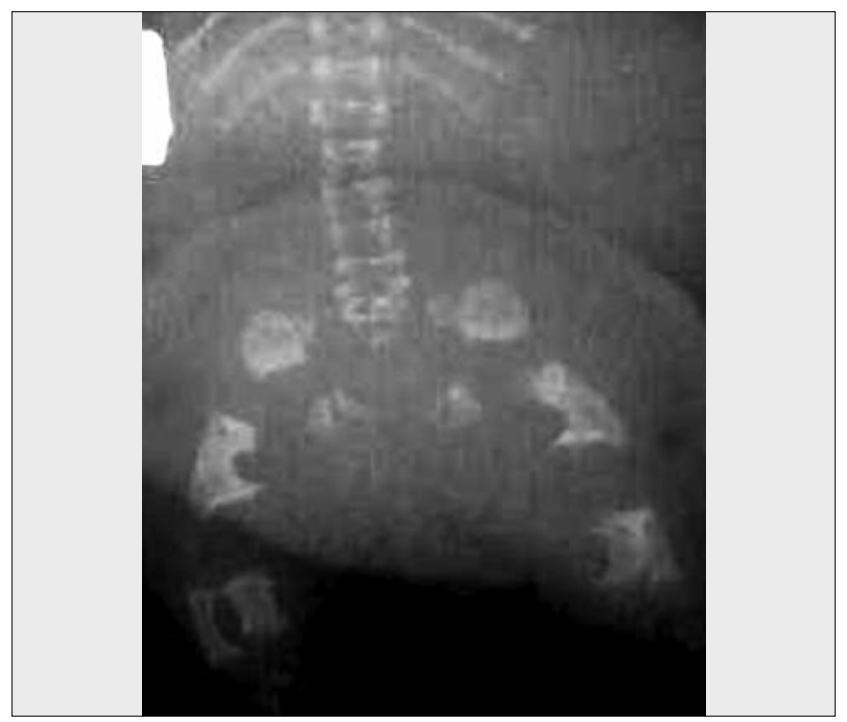

Figure 9. Schneckenbecken dysplasia. Note severe platyspondyly, thin ribs and snail-shaped iliac bones
(Figure 10), and loss of mandibular angle accompanied by wormian bones and acroosteolysis in pycnodysostosis (Figure $11 \mathrm{a}, \mathrm{b}, \mathrm{c})$ (7).

Step III (Differentiation of Normal Variants from Pathological Abnormalities): This last step requires experience in the field of pediatric radiology. It essentially involves recognition of normal variation from pathological abnormalities in the growing skeleton. Every portion of every bony structure should be looked at in an effort to combine the clinical, often dysmorphic findings previously noted in evaluation of the patient. Pathognomonic findings help to narrow the group of differential diagnosis leading to a specific entity.

At this point, having had a thorough clinical and radiographic assessment, even a simple radiographic grouping can be helpful to the clinician for the establishment of clinical care and follow-up. Table 2 provides a list of the grouping mentioned with common specific entities to consider (7). If a specific diagnosis cannot be made, it is expedient to send the case to a local expert, or an expert group in Skeletal Dysplasias such as the International Skeletal Dysplasia Registry at Cedars Sinai MC [muv.csmc.edu/skeletaldysplasia].

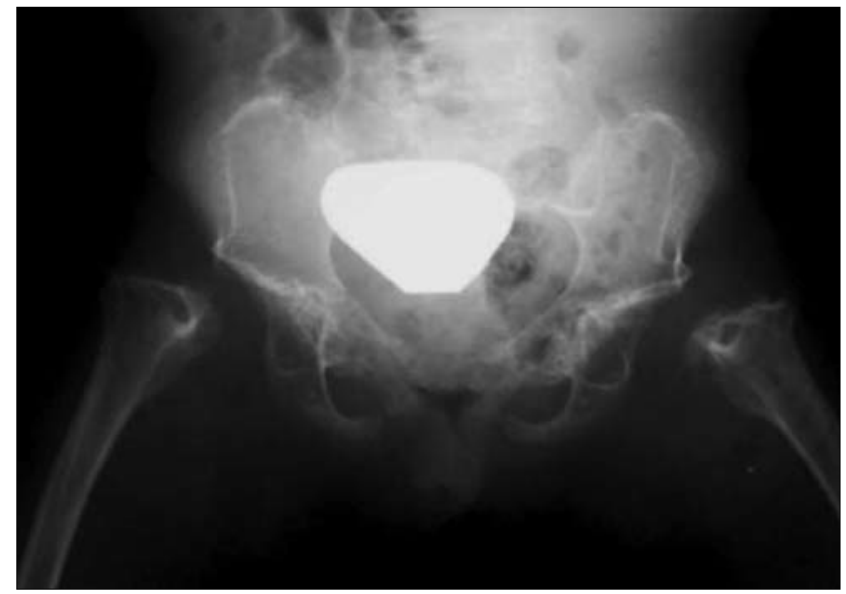

Figure 10. Dyygve-Melchior-Clausen syndrome. Note "lacy" iliac crest

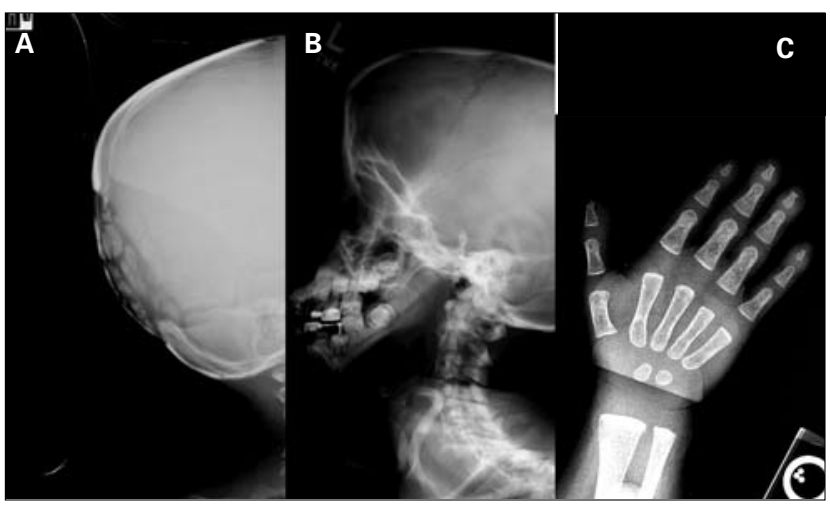

Figure 11. a,b,c. Pycnodysostosis. Loss of mandibular angle with wormian bones, large fontanelle and acroosteolysis in distal phalanges of hand 
Table 2. Clues for radiographic diagnosis of skeletal dysplasias

RADIOLOGICAL GROUPS COMMON ENTITIES RADIOLOGICAL FINDINGS

\section{Achondroplasia Group}

Thanatophoric
Dysplasia

Achondroplasia

Hypochondroplasia

Metatropic Dysplasia Group

Short-Rib Polydactyly Group

Short-Rib

(With or Without)-

Polydactyly

Dysplasia

Asphyxiating

Thoracic Dysplasia

(Jeune's syndrome)

Chondroectodermal

(Ellis van Creveld)

Dysplasia
Skull: Proportionately large skull, narrow skull base, kleeblattschadel Spine: flat, small vertebral bodies with rounded anterior ends Pelvis: small, flared iliac bones; very narrow sacrosciatic notches; flat, dysplastic acetabula Extremities: generalized micomelia: French telephone receiver femurs, round proximal femoral metaphyses with medial spike

Skull: enlarged, midface hypoplasia; rarely hydrocephalus, tight foramen magnum Thorax: small; shortened and anteriorly splayed ribs

Spine: slight platyspondyly, short and anteriorly round vertebral bodies that normalize from childhood on; very short pedicles with decreased interpedicular distance marked in lumbar spine; posterior vertebral scalloping that persists through life

Pelvis: flared, superiorly and laterally flattened ilia (elephant ear-shaped iliac wings), narrow sacrosciatic notches, flat acetabular roofs

Extremities: rhizo-, meso-, and acromelia

Hands: brachydactyly, metacarpal metahyseal cupping, phalangeal metaphyseal widening Knees: chevron and upside-down chevron deformity (tibia/femur) Hips: proximal femoral fade-out (infancy); hemispheric capital femoral epiphyses, short femoral necks

Legs: proximal and distal fibular overgrowth

Arms: prominent deltoid insertion area

The radiological findings are identical to achondroplasia but to a milder degree. All cases exhibit interpedicular narrowing in the lumbar spine. There may be brachydactyly, fibular overgrowth, short femoral necks. Other achondroplasia-like changes may or may not be present.

Thorax: small; short ribs

Spine: dense wafer vertebral bodies (newborn), platyspondyly (child, adult), scoliosis (adult) Pelvis and Hips: short, squared iliac wings; flat irregular acetabular roof; narrow sacrosciatic notches; halberd (hunting ax)-shaped proximal femurs

Extremities: trumpet-shaped metaphyses (newborn), dumbbell-shaped short tubular bones of hand and feet

Thorax: small; extremely short horizontal ribs

Spine: relatively normal

Pelvis: small, dysplastic ilia

Extremities: micromelia; round-ended femora; ovoid or tiny normal-shaped tibiae; severe brachydactyly with hypoplastic middle and distal phalanges; polydactyly common, not essential

Thorax: long and barrel shaped, handlebar clavicles, short horizontal ribs with bulbous anterior ends

Spine: normal

Pelvis: small; short, flared iiliac wings; trident acetabular roof; narrowed sacrosciatic notches Extremities: generalized shortening, precocious proximal femoral epiphyseal ossification, cone-shaped epiphyses in hand

Thorax: small, moderatley short ribs

Pélvis: small; short, flared iliac wings; trident acetabulas; narrowed sacrosciatic nothches Spine: normal

Extremities: generalized shortening with meso- and acromelia; premature ossification of capital femoral epiphyses, humeral and femoral bowing

Hands: characteristic-postaxial polydactyly, capitate/hamate (and other carpal) fusions, extra carpal boné, cone-shaped epiphyses

Feet: polydactyly 
Alanay $Y$ et al.

Radiological Evaluation of Skeletal Dysplasias

Table 2. (continued)

Diastrophic Dysplasia Group

\section{Diastrophic \\ Dysplasia}

MED-Multilayered Patellae/Brachydactyly/

Clubfeet

Type II Collagenopathies

Spondyloepiphyseal Dysplasia Congenita

Kniest Dysplasia

Other Spondylo-Epi-(Meta) Physeal Dysplasias

Spondyloepiphyseal Dysplasia Tarda

Dyggve-MelchiorClausen Syndrome

\section{Multiple Epiphyseal Dysplasia and}

Pseudoachondroplasia Group

Multiple Epiphyseal

Dysplasia

Pseudoachondroplasia

\section{Chondrodysplasia Punctata}

Group

Rhizomelic

Chondrodysplasia

Punctata
Head: ear pinna calcification

Thorax: moderately small

Spine: progressive scoliosis, kyphosis, odontoid hypoplasia, cervical kyphosis Extremities: micromelia; short, thck tubular bones; generalized barchydactyly-short ovoid first metacarpal, twisted metatarsal, accessory and irregular carpal bones; epiphyseal dysplasia, joint dislocations

Epiphyseal dysplasia especially at hips (half/quarter moon shaped) Double layered patella (lateral knee radiograph)

Mild brachydactyly

Clubfeet/ twisted metatarsals

Thorax: small; short ribs

Spine: oval vertebral bodies (newborn), anteriorly rounded platyspondyly (later)

Pelvis: absent pubic ossification (newborn and infancy)

Extremities: normally modeled but shortened long bones, significant generalized ossification delay (early) and hypoplastic/dysplastic epiphyses (later), unossified talus/calcaneus in the newborn, normal hands and feet with ossification delay

Thorax: small to normal

Spine coronal clefts (newborn/infancy), platyspondyly with end plate irregularity (later) Extremities: dumpbell femurs; generalized ossification delay, epiphyses becoming hypoplastic/dysplastic then megaepiphyses, cloudy effect in the physeal plate (late childhood); hand-bulbous joints (metaphyseal flare/epiphyseal fragmentation)

Spine: mild platyspondyly with centrally humped end plates with intervertebral disc space narrowing

Extremities: mild-moderate "epiphyseal dysplasia" (small and irregular epiphyseal centers), sparing hands and feet

Skull: microcephaly

Thorax: broad; anterior rib widening

Spine: double-humped vertebral bodies with end plate notching and posterior scalloping Pelvis: small iliac wings with irregularly calcifies "lacy" iliac crest

Extremities: moderate shortening with epi/metaphyseal changes, generalized brachydactyly with cone-shaped epiphyses and small carpal bones

Spine: young adult-disc herniations into vertebral end plates (Schmorl nodules)Hips: mistakenly diagnosed as bilateral Legg-Calve-Perthes disease or Meyer dysplasia Extremities: small, irregular, flattened ossification centers (epiphyses); small, irregular carpal (and tarsal) centers

Skull: normal

Thorax: mild anterior rib widening

Spine: superiorly and inferiorly rounded vertebral bodies, anterior central tongue, normalization of vertebrae (later)

Pelvis: rounded iliac wings, hypoplastic, poorly formed acetabular roofs

Extremities: mini-epiphyses in the hips, moderate-severe generalized epiphyseal dys plaisa (small, irregular, poorly ossified), metaphyseal widening and irregularity in the knees, proximally rounded metacarpals with mini-epiphyses in the hands, irregular carpal/tarsal bones

Spine: coronal clefting, anteriorly rounded vertebral bodies

Extremities: stippling, symmetric bilateral shortening of femurs (and humeri) with less severe shortening of long bones in general 
Alanay $Y$ et al.

Radiological Evaluation of Skeletal Dysplasias

Table 2. (continued)

Conradi-Hünermann

Syndrome/Dysplasia

Brachytelephalangic Chondrodysplasia

Punctata

\section{Metaphyseal \\ Chondrodysplasia Group}

Jansen-Type

Metaphysea

Chondrodysplasia

McKusick-Type

Metaphyseal

Chondrodysplasia

Schmid-Type

Metaphysea

Chondrodysplasia

Spondylometaphyseal

Dysplasia Group

\section{Mesomelic Dysplasia \\ Group}

Dyschondrosteosis

Acromelic/Acromesomelic Dysplasia Group

Dysplasias With

Prominent Membranous

Bone Involvement

Acromesomelic
Spine: diffuse stippling, scoliosis in childhood, abnormal vertebral body formation Extremities: mild symmetric or asymmetric shortening, diffuse generalized stippling in epiphyseal areas; hands and feet-normal aside from stippling ${ }^{* *}$ stippling resolves during infancy to develop normal or malformed epiphyseal centers

Spine: hypoplastic vertebral bodies with posterior scalloping and anterior rounding; stippling especially in the sacrococcygeal area

Extremities: normal length (mildly short), brachydactyly with hypoplastic tufts and deformed hypoplastic proximal phalanx of the second digit in the hand and first metatarsal of the foot

Skull: Brachycephaly, platybasia, small mandible

Thorax: normal size; expanded irregular anterior rib ends

Extremities: extensive irregularity of markedly expanded metaphyses- wide separation of epiphyses from metaphyses

** Cartilage-Hair Hypoplasia

Thorax: anterior rib widening/flaring

Spine: slightly small squared vertebral bodies

Extremities: flaring cupping fragmentation of metaphyses (especially knees), hips usually sparde; hands-marked shortening with metacarpal and phalangeal cupping and coning

Thorax: widening anterior rib ends

Spine: transient vertebral changes in middle childhood

Extremities: metaphyseal flaring, especially at the knees; rounded capital femoral epiphysis with widened growth plate; coax vara; usually no hand involvement

Spine: severe platyspondyly, anteriorly rounded/wedged vertebral bodies, increased intervertebral disc spaces, overfaced pedicles

Pelvis: short, flared iliac wings; irregular hypoplastic acetabular roof

Extremities: widening, sclerosis and irregularity of metaphyses; hemispheric capital femoral epiphysis and widened proximal femoral growth plate with irregularity on both sides; hands-mild shortening with metaphyseal cupping and irregularity, marked carpal ossification delay

Extremities: symmetrical bowing and shortenng of both radii, shortened ulnae, radiographic Madelung's deformity changes, variable tibial and fibular shortening

Trichorhinophalangeal Syndrome Type I and II Dysplasia of Maroteaux

Spine:oval vertebral bodies (early), anterior beaking and posterior wedging (later), gibbus and/or kyphoscoliosis ultimately

Extremities: shortening of all tubular bones, especially radius/ulna and tibia/fibula; very short tubular bones of hand and feet with cone-shaped epiphyses and large great toes

Skull: large, brachycephalic; wormian bones; wide sutures; persistently open anterior fontanelle

Thorax: absence/hypoplasia of clavicles, mildly shortened ribs with downward slope, 11 ribs Spine: significant posterior wedging of thoracic vertebrae

Pelvis: high narrow iliac wings, absence/hypoplasia of pubic bones

Extremities: numerous pseudoepiphyses of metacarpals and tapered distal phalanges in the hands 
Alanay $Y$ et al.

Radiological Evaluation of Skeletal Dysplasias

\section{Table 2. (continued) \\ Bent Bone Dysplasia \\ Group}

Campomelic

Dysplasia

\section{Dysostosis Multiplex}

Group

Dysostosis

Multiplex

Morquio's Syndrome

(MPS IVA, B)

Mucolipidosis II

(I Cell Disease)

\section{Dysplasias With Decreased Bone Density}

\section{Dysplasias With Defective Mineralization}

Increased Bone Density Without Modification of Bone Shape Group

\section{Osteogenesis}

imperfecta

type II, perinatal lethal

Osteogenesis

Imperfecta-other types

Hypophosphatasia

Osteopetrosis
Skull: enlarged, narrow with a small face

Thorax: mildly short ribs, 11 ribs; severe hypoplasia of the bodies of scapulae

Spine: nonossification of thoracic pedicles, cervical kyphosis, hypoplasia of cervical vertebral bodies

Pelvis: narrow, tall, iliac wings

Extremities: proportionately long, bowed femurs, short tibiae; short long bones of upper extremity

Skull: enlarged neurocranium, abnormal J-shaped sella

Thorax: short, thick clavicles; paddle (oar)-shaped ribs; hypoplastic glenoid

Spine: gibbus, superior notched (inferior beaked) thoracolumbar vertebral bodies, upper cervical subluxation

Pelvis: flared, small iliac wings with inferior tapering, steep acetabular roofs

Extremities: diaphyseal widening of long bones (marrow expansion); dysplastic epiphyses characteristic hand-brachydactyly, proximal metacarpal "pointing" diaphyseal widening of metacarpals and proximal/middle phalanges, small irregular carpal bones

Skull: no J-shaped sella

Thorax: widened, not oar shaped ribs

Spine: middle tonguing, not inferior beaking

Pelvis: no tapering of ileum

Extremities: proximal metacarpal rounding, not pointing, of hands

Extremities: severe osteopenia, poorly defined cortices, "periosteal cloacking" (newborn); rickets-like appearance in distal ulna and radius (infancy)

Dysostosis multiplex occurs later

Skull: very poor to no ossification

Thorax: small, narrow chest; beaded ribs

Spine: severe deossification, collapsed vertebral bodies

Skull: wormian bones (>8 to 10), variable decreased ossification

Spine: wedged or collapsed vertebrae

Remaining skeleton: osteoporosis and pathological fractures

Perinatal lethal/Infantile:

Skull: decreased ossification with single island-like centers for frontal occipital and parietal bones

Thorax: poorly ossified ribs; sporadic dropout of ribs; thin, wavy, fractured ribs

Spine: spoardc unossified vertebral bodies, dense and osteopenic vertebrae, butterfly shaped vertebral bodies

Extremities: generalized decreased ossification, chromosome-shaped femurs, metaphyseal cupping and irregularity, central luscent defect, bowed femora

* clavicles are not affected; infantile form is less severe

Adult

Generalized osteopenia

Extremities: metaphyseal widening (rickets-like chages), punched-out metaphyseal lesions, pathologic fractures

Generalized increased bone density

Skull: thick and dense, especially at the base

Thorax: splayed anterior ribs

Spine: "sandwich" vertebral bodies

Extremities: splayed metaphyses, bone-within-bone configuration, dense metaphyseal bands 
Alanay $Y$ et al.

Radiological Evaluation of Skeletal Dysplasias

Table 2. (continued)

\begin{tabular}{|c|c|c|}
\hline & Pyknodysostosis & $\begin{array}{l}\text { Generalized osteosclerosis } \\
\text { Skull: marked delay in closure of fontanelles and sutures, wormian bones, obtuse or } \\
\text { absent mandibular angle, dense skull } \\
\text { Thorax: resorbed acromial ends of clavicles } \\
\text { Extremities: resorbed phalangeal tufts }\end{array}$ \\
\hline \multicolumn{3}{|l|}{ Craniotubular Dysplasias } \\
\hline & $\begin{array}{l}\text { Craniodiaphyseal } \\
\text { Dysplasia }\end{array}$ & $\begin{array}{l}\text { Skull: marked thickening and sclerosis of calvarium and facial bones, obliteration } \\
\text { of foramina and sinuses } \\
\text { Thorax: diffusely widened, sclerotic ribs and clavicles } \\
\text { Extremities: straightened, undermodeled long bones diaphyses with metaphyseal } \\
\text { sparing; sclerosis (cortical thickening) of the short tubular bones of hands }\end{array}$ \\
\hline & Craniometaphyseal & $\begin{array}{l}\text { Skull: diffuse hyperostosis of cranialvault base and facial bone, obliterated } \\
\text { Dysplasia paranasal sinuses } \\
\text { Extremities: sclerosis of diaphyses (early), undermodeled flared metaphyses of } \\
\text { long bones (later) }\end{array}$ \\
\hline & Pyle Disease & $\begin{array}{l}\text { Skull: Mild skull and facial involvement, minimal base-of-skull sclerosis, prominent } \\
\text { supraorbital ridging } \\
\text { Thorax: mildly thick clavicles and ribs } \\
\text { Pelvis: thickened ischium and pubis } \\
\text { Extremities: marked undertubulation of long bones, especially distal femurs } \\
\text { (Erlenmeyer flask deformity); distal flaring of metacarpals and proximal flaring of } \\
\text { phalanges }\end{array}$ \\
\hline \multicolumn{3}{|l|}{$\begin{array}{l}\text { Disorganized Development } \\
\text { of Cartilagenous Bony and } \\
\text { Fibrous Components of the } \\
\text { Skeleton }\end{array}$} \\
\hline & Spondyloenchondrodysplasia & $\begin{array}{l}\text { Spine: severe platyspondyly with end plate irregularity } \\
\text { Extremities: enchondromata at distal and proximal ends of long bones, hands and } \\
\text { feet are rarely affected }\end{array}$ \\
\hline & Dysspondyloenchondromatosis & $\begin{array}{l}\text { Spine: vertebral anomalies, hemivertebrae, anisospondyly and end plate irregularity } \\
\text { Extremities: typical enchondromata, including hands and feet, with long bone } \\
\text { asymmetry }\end{array}$ \\
\hline \multicolumn{3}{|l|}{ Osteolysis Group } \\
\hline & $\begin{array}{l}\text { Multicentric Carpal/Tarsal } \\
\text { Osteolysis With or Without } \\
\text { Nephropathy }\end{array}$ & $\begin{array}{l}\text { Extremities (wrist and ankles): deossification of carpal bones, loss of carpal/tarsal } \\
\text { contours, bone resorption and collapse, sclerosis sometimes extending } \\
\text { into adjascent short tubular bones }\end{array}$ \\
\hline \multicolumn{3}{|l|}{ Patellar Dysplasia Group } \\
\hline & Nail-Patella Syndrome & $\begin{array}{l}\text { Spine: normal } \\
\text { Pelvis: iliac horn in the center of the iliac wing extending posteriorly } \\
\text { Extremities: (knees and elbows) hypoplastic or absent patella's, radial head and } \\
\text { capitellum hypoplasia/elbow dislocation }\end{array}$ \\
\hline
\end{tabular}

\section{Conclusion}

The complete group of osteochondrodysplasias, although individually rare, is an important group of disorders for healthcare providers who deal with individuals with short stature. These individuals present with significant morbidities due to destruction of bone and cartilage caused by defects in linear growth, bone modeling and regeneration. Regardless of the specific diagnosis, skeletal dysplasias in general share clinical and radiological findings helping us to group them in several ways. In this review, we aimed to focus on the radiological aspect of assessment of skeletal dysplasias. We also included an outline of the basic clinical approach to an individual with a suspected skeletal dysplasia. The recent advances in the field of molecular pathogenetic mechanisms underlying skeletal dysplasias are beyond the scope of this review. However, we would like to emphasize that accurate clinical, radiological and finally molecular diagnosis of skeletal dysplasias is more important than ever in this era of up-to-date genetic counseling, prenatal, preimplantation genetic diagnoses and hopefully, molecularly targeted therapeutics in the future. 


\section{Acknowledgement}

RSL, is supported by NIH GRANT\# HD 22657.

\section{References}

1. Orioli IM, Castilla EE, Barbosa-Neto JG. The birth prevalence rates for skeletal dysplasias. J Med Genet 1986:23:328-332.

2. Warman ML, Cormier-Daire $V$, Hall $C$, Krakow D, Lachman R, LeMerrer M, Mortier G, Mundlos S, Nishimura G, Rimoin DL, Robertson S, Savarirayan R, Sillence $D$, Spranger J, Unger S, Zabel B, Superti-Furga. Nosology and classification of genetic skeletal disorders: 2010 revision. Am J Med Genet 2011;155:943-968.
3. Unger S, Lachman RS, Rimoin DL. Chondrodysplasias. In: Rimoin DL, Connor JM, Pyeritz RE, Korf B (eds) Emery and Rimoin's Principles and Practice of Medical Genetics, 5th ed., vol. 3. Elsevier, Philadelphia, PA, USA, 2007;3709-3753.

4. Superti-Furga A, Unger S. Nosology and classification of genetic skeletal disorders: 2006 revision. Am J Med Genet A 2007;143:1-18.

5. Krakow D, Rimoin DL. The skeletal dysplasias. Genet Med 2010;12:327-341.

6. Mortier GR. The diagnosis of skeletal dysplasias: a multidisciplinary approach. Eur J Radiol 2001;40:161-167.

7. Lachman RS. Taybi and Lachman's Radiology of Syndromes, Metabolic Disorders and Skeletal Dysplasias, $5^{\text {th }}$ ed., Elsevier, Philadelphia, PA, USA, 2007. 